\title{
ILCEA
}

Revue de l'Institut des langues et cultures

d'Europe, Amérique, Afrique, Asie et Australie

$15 \mid 2012$

Les mots de la crise

\section{La crise financière de 2007-2009 à travers son lexique dans la presse américaine : caractérisations graphiques et enjeux de traduction}

The 2007-2009 financial crisis seen through its specialised lexicon in the American press: graphic characterisations and translation issues

\section{Christian Leblond}

\section{OpenEdition}

Journals

Édition électronique

URL : http://journals.openedition.org/ilcea/1151

DOI : 10.4000/ilcea.1151

ISSN : 2101-0609

Éditeur

UGA Éditions/Université Grenoble Alpes

Édition imprimée

ISBN : 978-2-84310-220-2

ISSN : $1639-6073$

\section{Référence électronique}

Christian Leblond, «La crise financière de 2007-2009 à travers son lexique dans la presse américaine caractérisations graphiques et enjeux de traduction », ILCEA [En ligne], 15 | 2012, mis en ligne le 30 janvier 2012, consulté le 01 mai 2019. URL : http://journals.openedition.org/ilcea/1151 ; DOI :

10.4000/ilcea. 1151

Ce document a été généré automatiquement le 1 mai 2019.

(C) ILCEA 


\title{
La crise financière de 2007-2009 à travers son lexique dans la presse américaine : caractérisations graphiques et enjeux de traduction
}

\author{
The 2007-2009 financial crisis seen through its specialised lexicon in the
} American press: graphic characterisations and translation issues

\section{Christian Leblond}

\section{NOTE DE L'AUTEUR}

L'auteur exprime ici sa gratitude envers les deux relecteurs anonymes de ce travail et tient à les remercier pour leurs observations et suggestions très constructives.

We have attained a degree of flexibility that can mitigate most significant shocks - a proposition as

yet not fully tested.

Alan GREENSPAN, 2005.

L'article qui suit constitue le premier élément d'un projet de recherche multilingue ${ }^{1}$ qui se donne pour objectif de mettre en évidence l'existence de «mots de la crise » dont la fréquence d'emploi au cours des mois de 2007-2009 est corrélée à l'évolution des indicateurs boursiers. Les données graphiques et chiffrées sur le nombre d'occurrences sont fournies par la base de recherche FACTIVA, selon une méthodologie précisée en introduction de ce volume (voir éditorial). La partie consacrée aux États-Unis ouvre le recueil car le point de départ de cette crise se situe clairement outre-Atlantique et les premiers éléments de lexique utilisés pour la décrire appartiennent à l'anglais américain avant d'être traduits ou adaptés aux autres langues européennes. En un certain sens, les données sur les États-Unis se situent en amont de l'étude traductologique dans les autres langues. Un soin tout particulier a donc été apporté dans cette première étude à la 
constitution d'une liste de termes dont la qualité de «mot de la crise » est vérifiée en formulant des hypothèses graphiques qui seront avérées ou non.

2 La structure de la présentation des termes dans l'étude ne reflète pas la chronologie de la recherche elle-même, mais le lecteur constatera qu'elle se calque sur une hiérarchie des résultats ex post. Dans le même mouvement, une étude traductologique sera conduite pour fournir des éclaircissements non pas sur la nature de la crise $^{2}$ mais sur les enjeux spécifiques - souvent extra-linguistiques - qui rendent la traduction non pas impossible mais périlleuse.

\section{Définitions et cadrage temporel}

Le mot « crise » est sans doute l'un des termes préférés des auteurs de sciences humaines, avec les mots "contraste » ou "paradoxe ", mais toute chute, tout dysfonctionnement n'est pas une crise. Si l'on représente graphiquement un phénomène en fonction du temps, une crise est une période relativement courte dans laquelle on entre par un point d'inflexion, moment où des tendances longues commencent à s'inverser, et dont on sort par un point de rupture qui marque nettement un avant et un après. Du point de vue épistémologique, une crise est une sorte de sas temporel, une période de rétrécissement qui correspond au seuil d'un nouveau territoire permettant de passer d'un paradigme ${ }^{3}$ à un autre. Une révolution scientifique au sens de Kuhn comporte ainsi une période de crise quand le paradigme dominant n'arrive plus à rendre compte de la totalité des phénomènes observés. La survenue d'une anomalie signale que la représentation qui fait l'objet d'un consensus sera bientôt dépassée et fera place à un dispositif plus puissant. La crise épistémologique est donc, par excellence, le moment de la transition, celui où les certitudes de la période antérieure sont dépassées, et où l'on attend l'avènement du nouveau modèle.

En économie, une telle définition de la crise ne serait pas très convaincante. En effet, ce qui fait passer d'une période à une autre serait plutôt désigné par le mot "révolution » tandis que les crises ne sont que les ratés récurrents d'une machine capricieuse pour laquelle il n'existe pas de remplaçant. La révolution industrielle - ou la révolution des technologies de l'information - frappe d'obsolescence le modèle économique et social qui avait jusqu'alors prévalu. Mais une crise économique ne possède pas en soi le pouvoir de faire accoucher nos sociétés d'un monde nouveau. En effet, depuis les travaux de Kondratieff, Kutznets Juglar et Kitchin ${ }^{4}$, il est admis que l'économie de marché n'évolue pas de manière linéaire mais cyclique, avec des périodes de récession et quelquefois de dépression ${ }^{5}$ qui sèment le doute, voire la panique, mais qui ne l'empêchent pas de perdurer.

5 Pour que le cycle des conjonctures alternativement hautes et basses débouche sur un changement radical, il faut postuler la probabilité d'une "grande» crise finale qui dépasse, par son ampleur et sa durée, les valeurs habituelles ${ }^{6}$. Mais en dehors de cette crise bien particulière et quelque peu hypothétique, il faut se résoudre à voir les années de vaches maigres succéder aux années de vaches grasses ${ }^{7}$, et renoncer à passer de l'âge de fer à l'âge d'or ou aux lendemains qui chantent.

6 Du point de vue linguistique, il nous semble encore plus hasardeux de parler de crise au sens eschatologique évoqué précédemment. Le langage humain s'enracine dans l'histoire, et connaît des changements très lents, fournissant une sorte de basse continue aux 
révolutions esthétiques et littéraires qui se jouent sur des scènes très étroites. Pourtant, les langues évoluent, et chaque période, dans le répertoire du lexique, puise ses mots de prédilection et invente de nouveaux termes, ainsi que de nouvelles combinaisons, si intimement liées aux années qu'elles peuvent en devenir les marqueurs les plus clairs.

7 Ainsi, dans le cadre du projet décrit, ce premier article a pour objectif d'étudier les correspondances entre un phénomène économique cyclique et un phénomène langagier dont nous aurons à déterminer les caractéristiques dans la presse des États-Unis. Nous testerons l'hypothèse selon laquelle il est possible d'isoler, pour la crise de 2007-2009, un appareil lexical unique et caractéristique qui constitue le versant langagier original et nous pourrions presque écrire "à usage unique», d'une phase récurrente du cycle économique.

Pour éviter de poser des hypothèses sur les lendemains de la crise et son impact sur les mentalités, nous adopterons une caractérisation purement morphologique, en appelant " crise », par la suite, une période du cycle des affaires - tel que les indicateurs boursiers permettent de le saisir - qui est celle de la chute du sommet vers les valeurs les plus basses. Autrement dit, si le cycle est représenté par une sinusoïde (quand la valeur de l'indice en fonction du temps est de type trigonométrique), la crise correspond pour nous à la demi-période centrée sur le moment où l'indice passe conventionnellement par la valeur zéro.

Figure 1. - Crise : peak to trough.

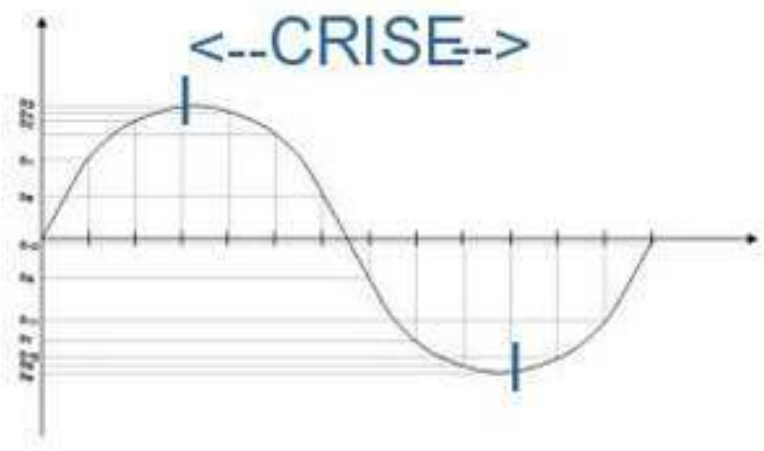

Figure 2. - L'indice Dow Jones, 2006-2010.

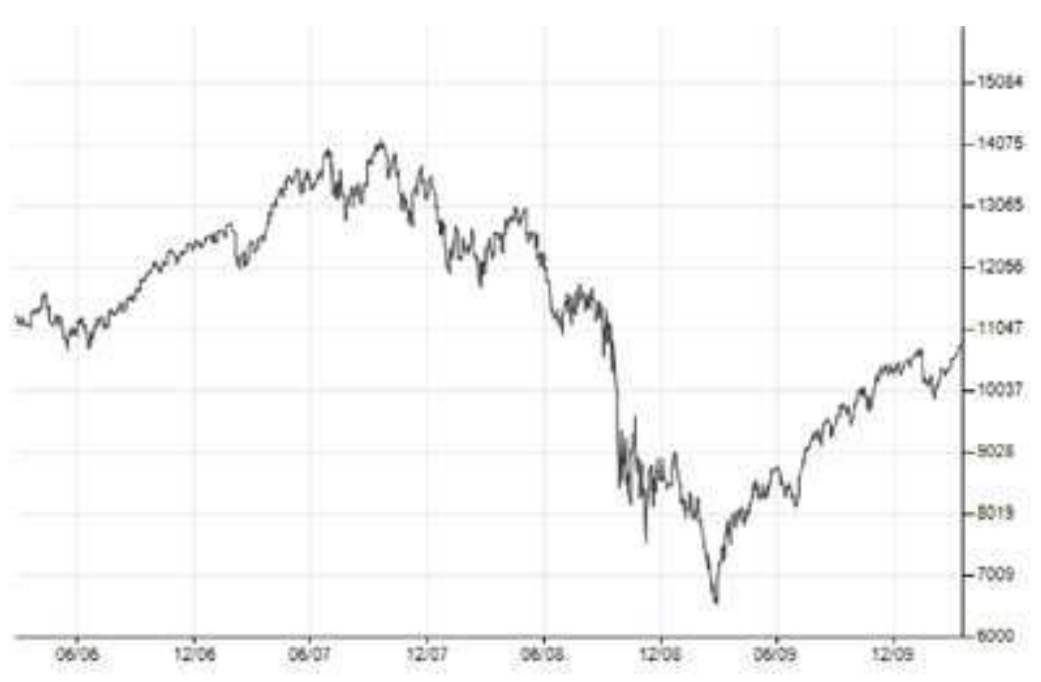


Cette définition n'a de sens réel que dans une description de phénomènes parfaitement cycliques que l'observation des fluctuations réelles ne permet pas de distinguer aussi nettement. Mais elle est suffisamment opérationnelle pour permettre d'aborder la phase suivante qui est celle du cadrage chronologique. L'autre intérêt de cette approche réside dans le fait qu'elle rendra possible une observation parallèle des indices et des mots de la crise dans la suite de l'étude.

La première phase de la crise se manifeste dès l'été 2007 par des turbulences sur les marchés financiers et se prolonge avec la faillite de Lehman Brothers, le 15 septembre 2008. Il s'ensuit une chute prolongée, jusqu'au mois de mars 2009, avant un redressement des cours qui caractérise la fin de l'année.

11 Strictement parlant, nous avons donc un peu débordé de la période de crise, car la variation sur les années 2007-2009 correspond à un peu plus d'une demi-période. Deux considérations nous ont guidés dans ce choix. Tout d'abord, le paramétrage de la base de données Factiva est facilité par des périodes annuelles entières. En outre, il faut pouvoir mesurer un éventuel décalage temporel entre le pic de fréquence du terme repéré et les fluctuations du marché.

Figure 3. - Le cadre chronologique : évolution du Dow Jones.

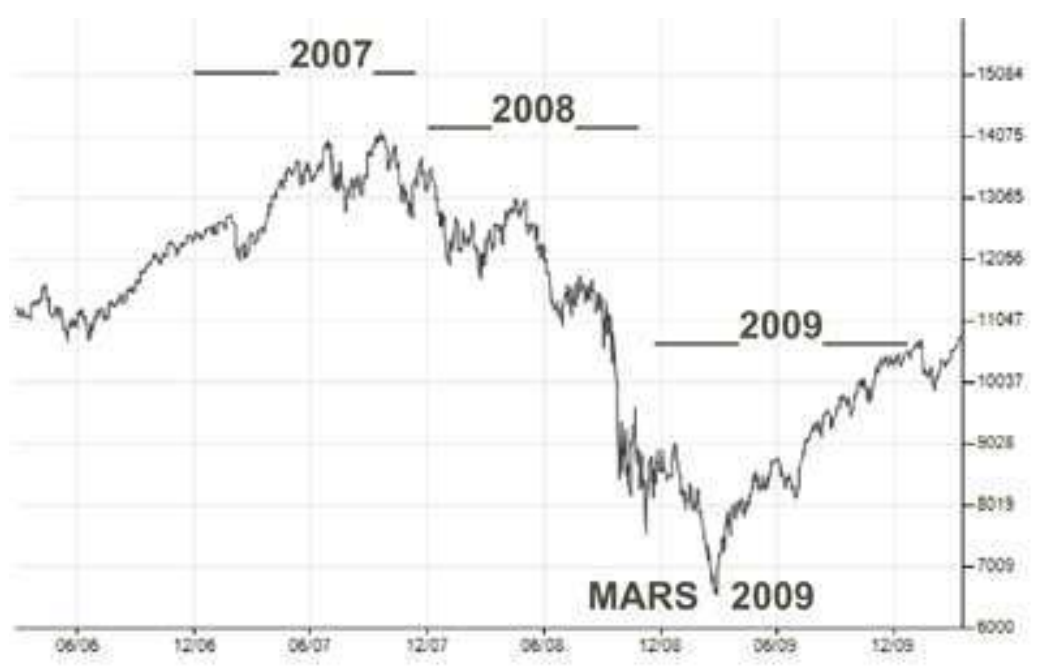

\section{Caractérisation graphique et constitution de la liste}

Si nous cherchons à mettre en évidence les termes et les expressions typiques de la crise, nous pouvons définir trois critères quant à la distribution de ses occurrences dans la période d'études précédemment définie. Un authentique mot de la crise doit, en toute logique, être lié de manière univoque à la période, c'est-à-dire :

- Être un terme (ou une expression) non attesté ou d'un usage très rare avant la crise ;

- Connaître un pic d'emploi statistiquement mis en évidence pendant la crise ;

- Son usage doit connaître un déclin rapide après la crise.

Dans le graphique $\mathrm{n}^{\circ} 4$, le lecteur trouvera l'histogramme généré par Factiva sur la période 2002-2010 pour le terme bubble qui semble a priori lié étroitement à la crise. Chaque colonne correspond à une année calendaire. Le graphique met en évidence que plusieurs des trois critères ne sont pas conformes au modèle. 
Figure 4. - Distribution des occurrences de bubble sur la période 2002-2010.

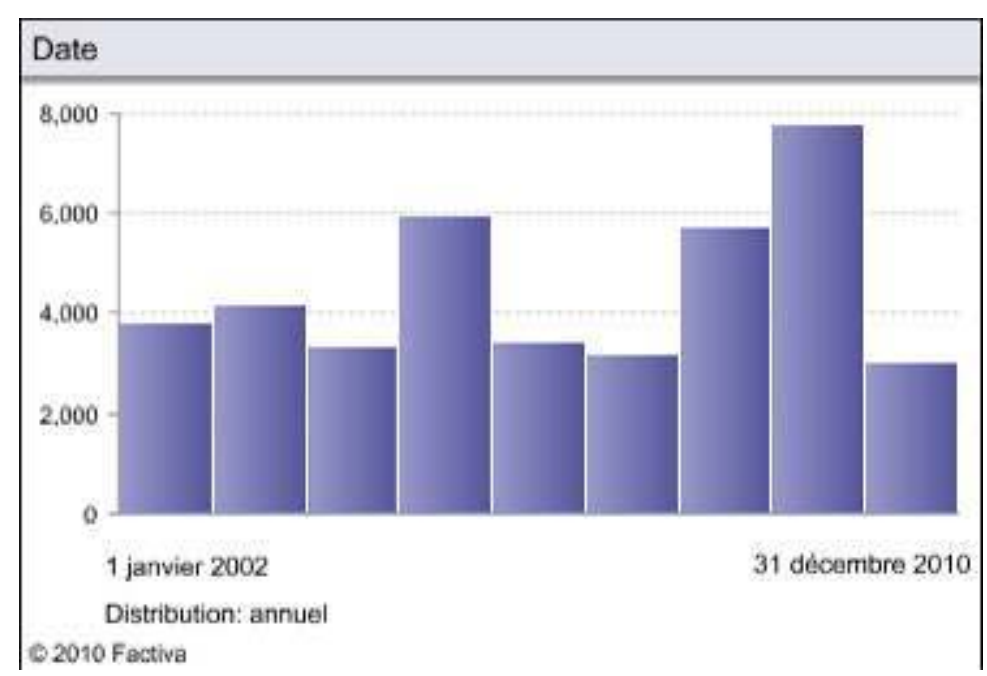
fréquence de 2007 est égale à celle de 2010 et celle de 2008 à celle de 2005. Le terme en question, bubble (bulle), a peut-être conceptuellement une valeur pour décrire le phénomène mais il n'est pas un «mot de la crise " au sens où on l'a défini. Il s'intègre vraisemblablement au bruit de fond langagier des crises, et en ce sens, le terme bubble est sans doute un mot des crises, mais pas un mot de la crise que nous étudions.

Le lecteur trouvera en revanche, à la figure 5, un graphique qui semble avoir été fait sur mesure pour constituer un cas d'école.

Figure 5. - Distribution du terme subprime sur la période 2002-2010.

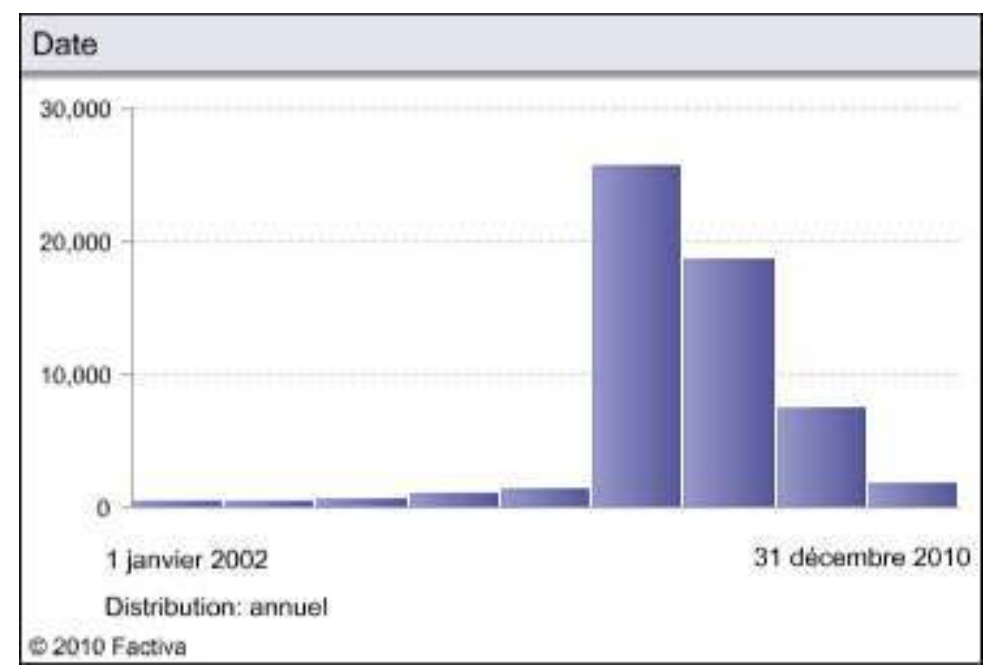

Pourtant ce graphique n'est pas une construction, il décrit la fréquence du terme subprime, pratiquement inexistant entre 2002 et 2006, et qui surgit verticalement en 2007 puis retombe rapidement dans l'oubli pour disparaître pratiquement de la presse vers 2010. Le taux d'occurrence est cependant non nul ce qui pourrait s'expliquer par des " faux positifs ${ }^{8}$ » assez improbables dans la mesure où le paramétrage de Factiva élimine du corpus les textes non économiques. En fait, l'examen détaillé montre que le terme était 
connu et utilisé par les spécialistes, mais qu'il ne devient d'usage courant qu'à la faveur de l'explosion de la bulle qui passera à la postérité sous le titre de " crise des subprimes ».

Les conséquences de nos choix méthodologiques commencent à se faire jour à ce stade. En définissant le mot de la crise d'après des critères purement extérieurs et objectifs, nous adoptons une démarche très différente de celle des économistes. En économie, la description du mécanisme explicatif implique une collecte sélective de données, voire un tri conceptuel, qui préempte en grande partie les interprétations que l'on est amené à formuler in fine. Autrement dit, sélectionner les termes qui font sens pour les économistes aboutirait vraisemblablement à des conclusions sur les mots clés de la crise qui, du point de vue du linguiste, seraient circulaires.

Pour mettre en évidence la similarité des phénomènes de fréquence et l'évolution des indices, nous pouvons faire pivoter le graphique déjà utilisé du Dow Jones, afin de pouvoir observer directement des morphologies ressemblantes?.

En effet, on peut s'attendre à ce qu'au plus fort de la crise, matérialisé par le point le plus bas de l'indice des valeurs boursières, la fréquence d'emploi des mots de la crise soit à son maximum.

Figure 6. - Image en miroir de l'indice Dow Jones.

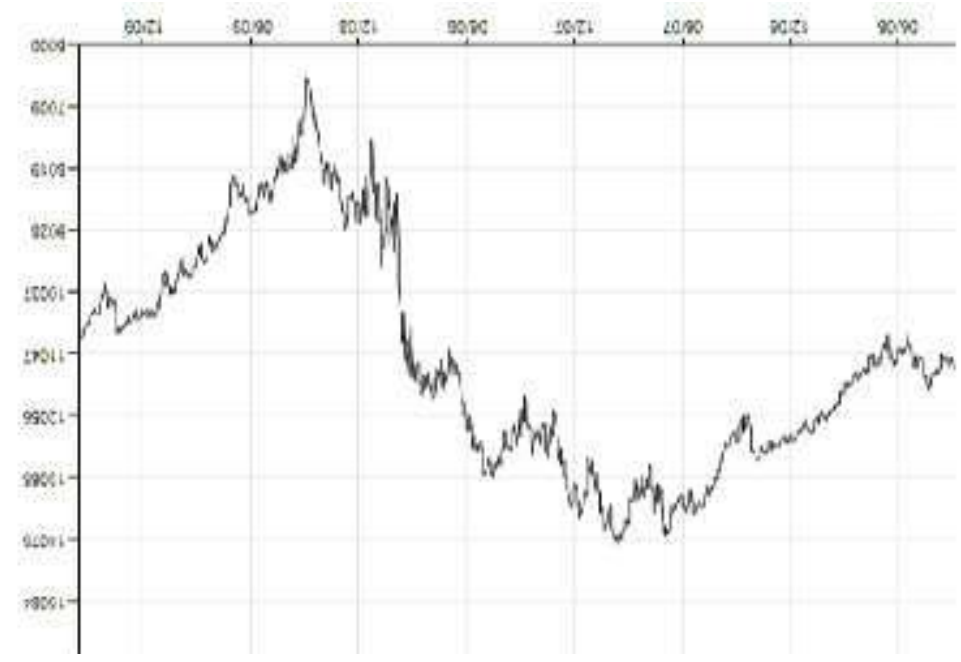


Figure 7. - Distribution du terme subprime (comparé au Dow Jones inversé).

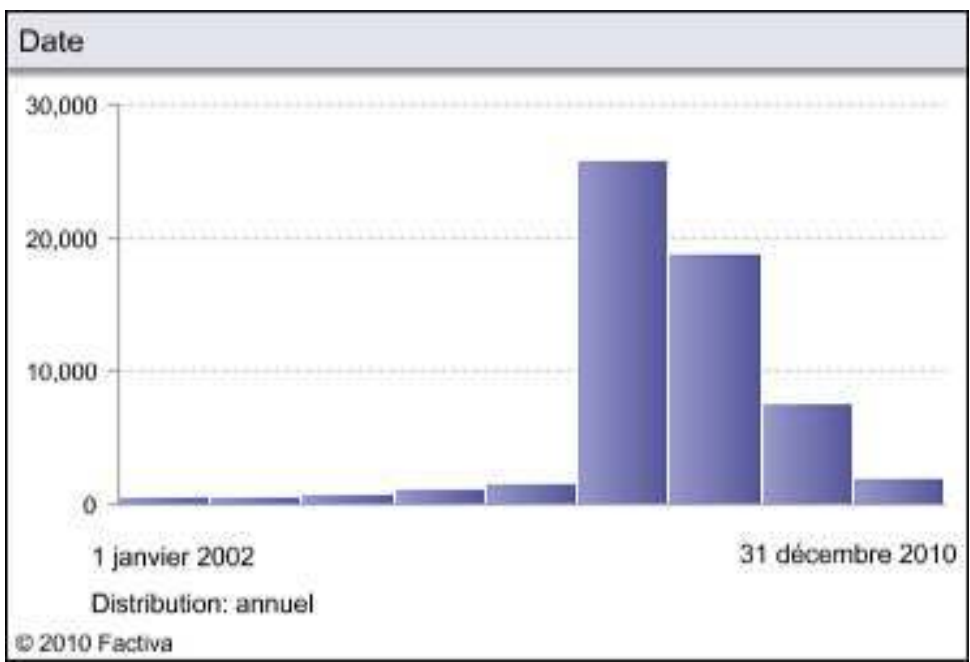

Plutôt que de mettre en équations cette hypothèse du "miroir ", nous estimons assez probant de juxtaposer deux tableaux Factiva pour les présenter directement au lecteur. Ce qui ressort de cette observation, c'est la corrélation temporelle de la fluctuation des deux valeurs. À partir de cette confrontation des séquences chronologiques, nous pouvons estimer que nous avons, au moins provisoirement, mis au point un cadre méthodologique qui va maintenant être utilisé comme banc d'essai des mots de la crise. Nous n'allons pas proposer au lecteur de lire en détail le protocole de la constitution de notre lexique de la crise mais, comme c'est souvent le cas, lui en présenter brièvement les grandes étapes. La première phase est une collecte, débouchant sur une masse foisonnante de termes :

- Lecture de la presse économique

- Repérage des thèmes récurrents

- Repérage des néologismes

La seconde phase est une phase de tri qui consiste à utiliser la méthode décrite, c'est-àdire rentrer dans un formulaire Factiva bien paramétré les termes les plus prometteurs, et tester leur conformité au modèle, retenir les plus intéressants pour les classer par degré de conformité :

- Test de validation

- Hiérarchisation

La liste à laquelle nous sommes parvenus est la série de termes anglais suivants que nous faisons suivre de leur traduction française quand elle existe et d'un commentaire entre parenthèses dans le cas contraire :

\begin{tabular}{|l|l|}
\hline$\cdot$ Recession & $\cdot$ récession \\
\hline$\cdot$ (housing) bubble & $\cdot$ bulle (immobilière) \\
\hline$\cdot$ Mortgage & $\cdot$ prêt immobilier \\
\hline$\cdot$ Subprime & $\cdot$ (dont le credit rating est négatif) \\
\hline$\cdot$ Foreclosure/Repossession & $\cdot$ saisie immobilière \\
\hline
\end{tabular}




\begin{tabular}{|l|l|}
\hline - Securitization & - titrisation \\
\hline - Bad bank & $\cdot$ Structure de défaisance \\
\hline - FDIC Federal Deposit Insurance Corporation & $\bullet$ (fond de garantie des banques) \\
\hline - Delinquency / Default & $\bullet$ défaut de paiement \\
\hline - Toxic assets (TARP : troubled assets recovery plan) & $\bullet$ actifs toxiques \\
\hline - SWAP/CDS (credit default swap) & $\bullet$ (produits dérivés d'assurance) \\
\hline - Hedge fund & $\bullet$ fond de couverture / fond spéculatif \\
\hline
\end{tabular}

\section{Histogrammes des termes ne présentant pas de problème de traduction}

Le mot recession (figure 8) est peut-être le seul qui soit à ce point récurrent et commun à toutes les crises. Sans surprise, nous observons une dispersion importante, et sur le début de la période, un premier pic signale l'éclatement de la dot com bubble (bulle Internet) en 2002. Là encore, mot des crises plutôt que mot de la crise de 2007-2009, il reflète l'intensité de la destruction de valeur sur la période, et peut être considéré comme un marqueur quantitatif qui confère à notre période un label de crise en l'inscrivant dans une série, mais qui ne permet en rien de délimiter sa singularité.

Figure 8. - Recession.

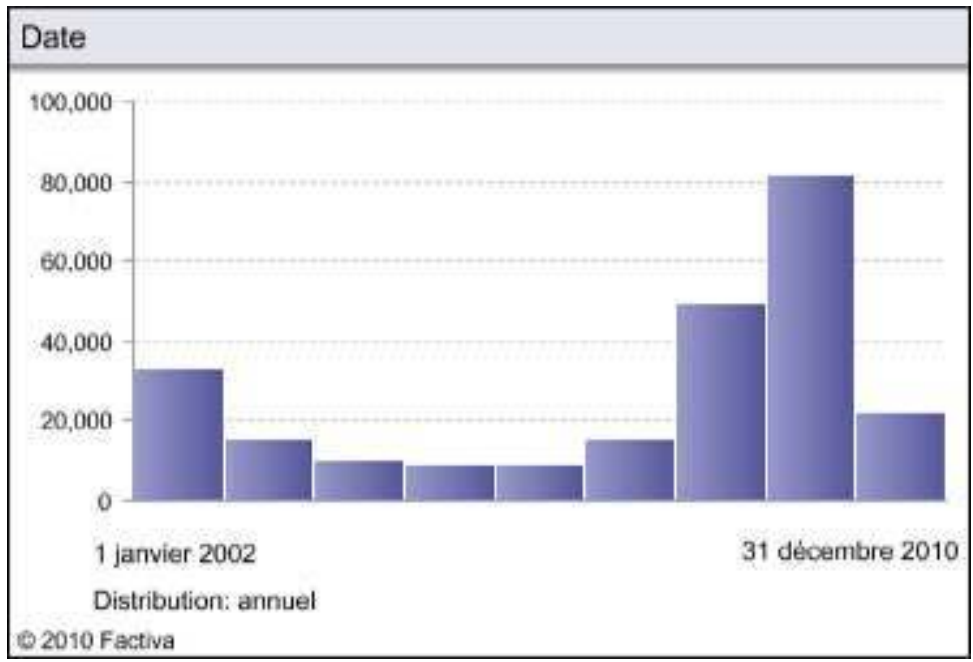

Le terme bubble (bulle, voir figure 9), dont la distribution sur la majeure partie de la décennie évoque encore plus le bruit de fond que la distribution précédente, affiche bien deux pics significatifs en 2003 et 2009. Il est intéressant à cet égard de noter que les deux maxima en question sont légèrement décalés temporellement par rapport au déclenchement des crises. Cette observation recoupe l'idée très souvent exprimée selon laquelle une bulle ne se reconnaît qu'à son éclatement. Dans un premier temps, un marché haussier est compris comme un signe de bonne santé économique. 
Figure 9. - (Housing) bubble.

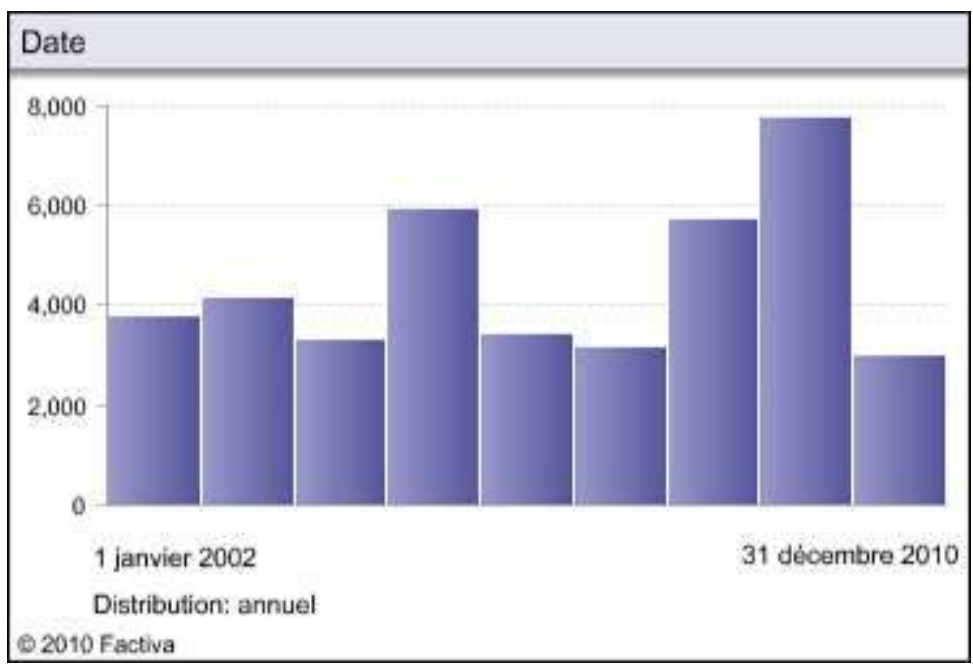

L'optimisme haussier accélère l'afflux de fonds qui cherchent à se placer et soutient les cours jusqu'au moment presque impossible à prédire où la plupart des opérateurs identifient la contradiction entre l'envolée des cotations et l'absence de fondamentaux tels que les perspectives de profits et, par conséquence, de dividendes et plus-values. Le cheminement entre cette prise de conscience et le retournement - le moment où le marché va massivement détruire de la valeur en révélant par là-même que celle-ci était illusoire - correspond aux derniers instants avant l'effondrement des cours. Pour ces raisons, le graphique de la distribution nous semble tout à fait révélateur et présente un intérêt pour le lecteur. Mais l'année 2005 accuse une montée tout à fait comparable en intensité avec l'année 2008. Pour cette raison, nous ne pouvons pas classer le terme bulle, pourtant capital dans la description du phénomène, dans le groupe des mots de la crise.

Figure 10. - (Mortage-backed) securities.

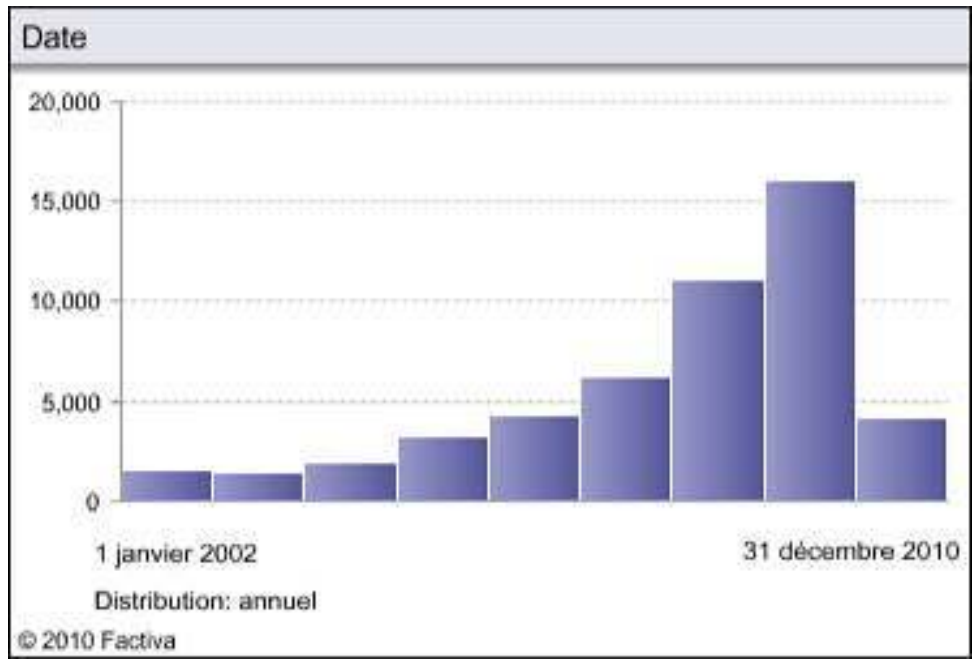

Le point de départ de la crise de 2007-2009, tous les observateurs s'accordent sur ce point, se trouve dans les excès du marché immobilier aux États-Unis. La partie inédite de la séquence immobilière est liée à une série d'innovations financières qui ont permis aux détenteurs de créances de repousser la date à laquelle les positions spéculatives doivent 
se dénouer. La distribution présentée par le graphique 10 démontre de manière assez convaincante que le composé mortgage-backed security (ou asset-backed security) possède une distribution qui corrobore presque parfaitement son statut de mot de la crise. Constatation complémentaire, l'expression est difficilement traduisible, contrairement au terme bubble, non seulement en tant que composé, mais également au niveau des composants. Le premier terme, mortgage, malgré ses origines évidentes dans le droit médiéval français, n'est qu'imparfaitement traduit par « crédit immobilier ».

Ces crédits «hypothécaires » sont la règle aux États-Unis, donc du point de vue de la fréquence d'emploi, un terme français banal est une solution satisfaisante. Les hypothèques ne sont pas rares en France, mais ce qui assure à l'organisme de crédit que l'emprunteur ne mettra pas la clé sous la porte, c'est la possibilité de saisir les rémunérations du mauvais payeur à la source. Aux États-Unis, les prêts hypothécaires sont des créances détenues par l'établissement de crédit, en général des banques ou des quasi-banques pour lesquelles c'est le bien immobilier lui-même qui sert de garantie si les emprunteurs font défaut.

Il n'y a pas de lexique spécialisé lié à ces mécanismes qui permettent souvent à l'emprunteur de "rendre les clés à la banque " pour solde de tout compte. Nous en voulons pour preuve que le terme non recourse ${ }^{10}$ n'est pas compris ni traduit dans la presse française. Mais la garantie hypothécaire peut s'avérer insuffisante pour couvrir la créance détenue par la banque si le marché de l'immobilier venait à se retourner. Pour cette raison, les banques se sont très souvent dessaisies de ces titres, et les ont revendues à des institutions plus à même d'amortir le risque de défaut en pratiquant une stratégie de levier plus prudente (c'est-à-dire, en gardant un ratio raisonnable entre encours des prêts et fonds propres).

En revanche, les Credit Default Swaps, terme non traduit, est compris des spécialistes et repris sous forme de l'acronyme CDS dans la presse d'affaires.

Figure 11. - SWAP - credit default swap.

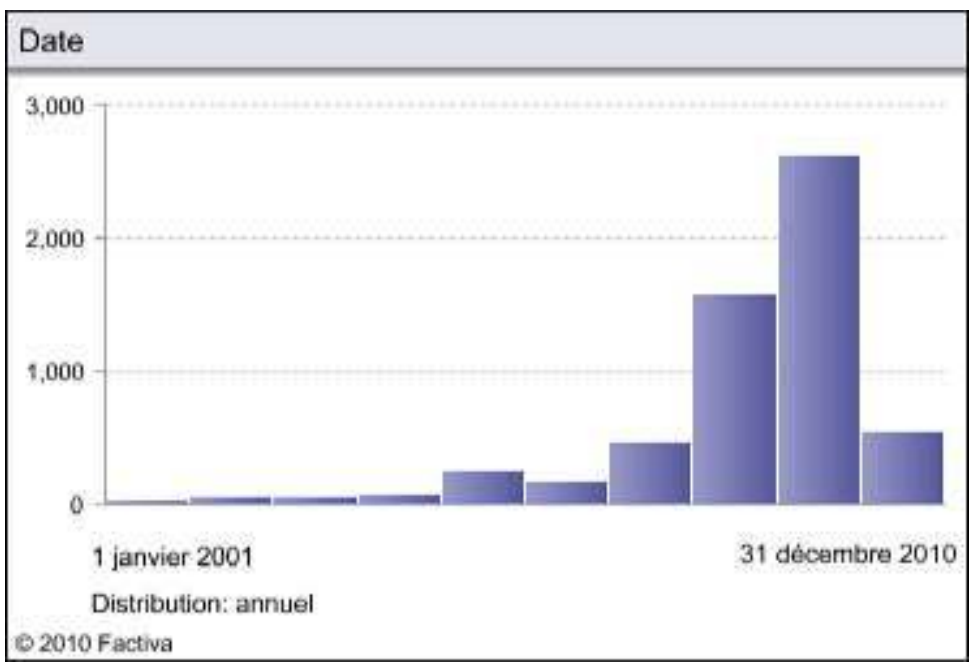

31 À partir des années 1980, celles de la dérégulation reaganienne, les produits dérivés sont présentés comme la solution miracle permettant de mettre l'État et les régulateurs sur la touche pour passer à un nouveau paradigme dans lequel, pour simplifier, le produit dérivé, instrument de marché, joue le rôle de stabilisateur autrefois assuré par la régulation. Le CDS est conçu comme un produit d'assurance avant de devenir un 
instrument de pure spéculation. Un swap permet, à l'origine, de se prémunir contre les mauvais payeurs, mais il est souscrit de façon prépondérante à la veille de la crise par des acteurs qui prennent des positions sur le risque de défaut de telle ou telle partie de la population sans détenir personnellement les biens immobiliers « assurés ».

\section{La règle de l'euphémisation}

Si l'on veut esquisser, à partir de ces exemples, une typologie dominante des termes les plus fréquents aux États-Unis, nous observons presque toujours une euphémisation du risque. Alan Greenspan, qui est à ce titre tout à fait emblématique de la période, fait preuve d'un optimisme modéré sur le chapitre de l'endettement immobilier de ses compatriotes :

In summary, although some broader macroeconomic measures of household debt quality do not paint as favorable a picture as do the data on loan delinquencies at commercial banks and thrifts, household finances appear to be in reasonably good shape. There are, however, pockets of severe stress within the household sector that remain a concern and we need to be mindful of the difficulties these households face. (Greenspan, 2004)

Passé maître dans l'art de la litote, le gouverneur de la FED livre ses propos en étant pleinement conscient qu'ils seront décryptés comme des prophéties. ${ }^{11}$ Mais le lecteur est en droit d'en douter, car l'avertissement final sur les "poches soumises à un stress sévère » n'a apparemment pas été pris en compte.

$\mathrm{Au}$ terme de cette partie de l'analyse, nous formulons l'hypothèse complémentaire concernant le mot de la crise: typiquement, c'est un terme composé qui n'est pas traduit en français. ${ }^{12}$ Cette absence de traduction assez systématique peut constituer le point de départ d'un questionnement.

Le terme anglais est conservé tel quel, dans une première hypothèse, parce que traduire est inutile dans un domaine où seuls les «spécialistes » s'intéressent aux arcanes. Une autre explication pourrait également s'appuyer sur le manque de recul temporel. En d'autres termes, les journalistes économiques n'auraient pas « eu le temps nécessaire » pour inventer une traduction française qui retienne l'attention du public. Mais un retour au tableau de distribution aux États-Unis en fonction du temps infirme assez facilement ces deux hypothèses. Le pic de fréquence se situe en 2009, c'est-à-dire à la fin de la période d'étude, après deux années de progression régulière. L'argument de l'absence de recul et de la faible diffusion n'est donc pas soutenable.

Il reste par conséquent à explorer une autre voie: la traduction est d'autant plus impossible qu'elle serait indispensable. Nous entendons par là que les termes utilisés sont suffisamment problématiques pour que le traducteur ne puisse pas faire son travail sans opérer un choix radical entre une neutralité technique et la levée des ambiguïtés. Ne pas traduire est un cas extrême de la première option - le traducteur devient presque superflu dans cette situation poussée jusqu'à l'absurde, tandis que traduire en explicitant revient à prendre parti, ce qui n'entre pas dans les attributions du traducteur. 
Figure 12. - Securitization.

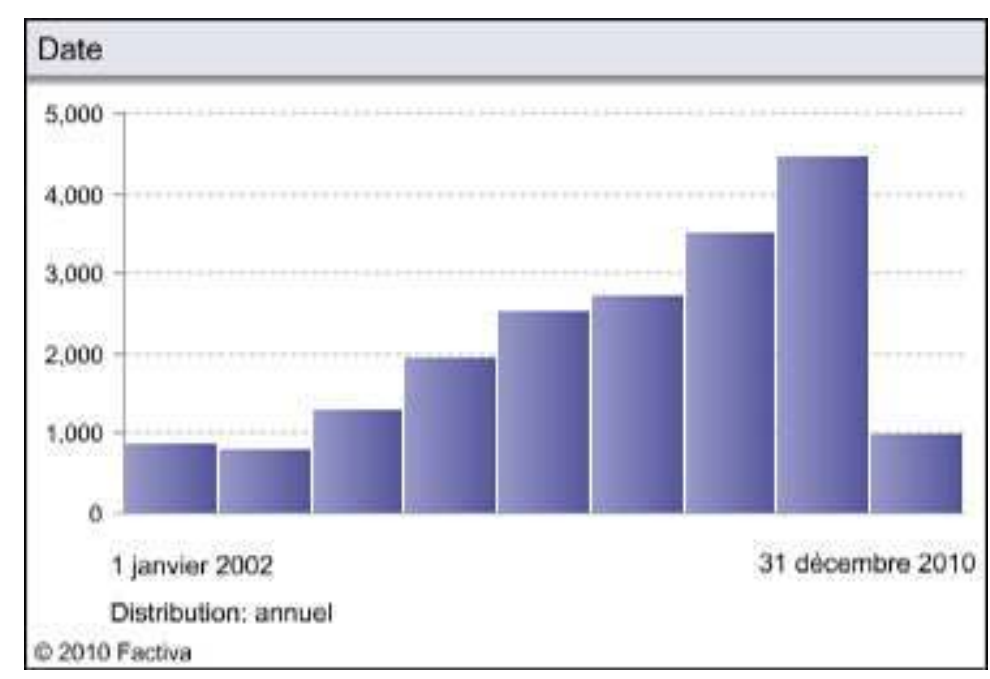

Le néologisme securitization (titrisation) passe positivement le test du graphique de distribution (figure 12). Ce terme qui décrit les nouveaux instruments spéculatifs apparus avant la crise, comme ceux du type mortgage-backed security ou credit default swap, est à la fois opaque et euphémistique. À la limite, les lecteurs anglophones auraient besoin d'une traduction en anglais courant (plain English) au même titre que les lecteurs francophones, car les enjeux de la traduction se sont déplacés vers l'amont. Le temps de la traduction serait alors le temps de la prise de conscience du risque réel, à travers les faux-semblants de la formulation par des euphémismes qui jouent sur les connotations positives des termes combinés. Les mots de la crise, tels que nous les circonscrivons à ce stade, confirment leur parenté évidente avec les discours extraordinairement lénifiants d'Alan Greenspan quelques années avant la crise :

These increasingly complex financial instruments have contributed to the development of a far more flexible, efficient, and hence resilient financial system than the one that existed just a quarter-century ago. (Greenspan, 2005)

Ces quelques lignes, assez représentatives du discours dominant en 2005, permettent de saisir pourquoi les investisseurs, lecteurs de la presse économique, ont accordé leur confiance aux produits dérivés sans en mesurer réellement les dangers. Le principal enjeu était de placer les termes nouveaux, d'une technicité un peu rebutante, dans la bonne catégorie, celle de la compétence, du progrès, de la sécurité et de l'assurance, en jouant sur les connotations positives de termes tels que securitization, CDS, et subprime qui soulignaient les limites de la prise de risque. Il est d'ailleurs indéniable que la vocation première des produits dérivés était de protéger l'investisseur contre les variations par trop erratiques des marchés.

Il est difficile de ne pas opérer un rapprochement entre cette pratique langagière et la manière dont, techniquement, la titrisation (securitization) a opéré un brouillage généralisé des pistes, pour ne pas dire une inversion du signifié. Sous la plume d'Alan Greenspan, une fois de plus, nous trouvons un véritable florilège des mots-clés de la révolution financière :

Conceptual advances in pricing options and other complex financial products, along with improvements in computer and telecommunications technologies, have significantly lowered the costs of, and expanded the opportunities for, hedging risks that were not readily deflected in earlier decades. The new instruments of risk 
dispersal have enabled the largest and most sophisticated banks, in their creditgranting role, to divest themselves of much credit risk by passing it to institutions with far less leverage. (Greenspan, 2005) avantages d'un système financier mondial basé sur le «privilège exorbitant » du pays central - les États-Unis - dans la mesure où il n'est que l'envers du « devoir exorbitant » qui attend ce même pays en temps de crise. la littérature économique, que les États-Unis de l'après-guerre jouent le rôle d'assureur de fait du système mondial au moment des crises. Selon eux, le fait que les États-Unis ne soient plus le «banquier du monde » mais le venture capitalist (investisseur en capitalrisque) du monde n'est pas inquiétant mais au contraire source de stabilité parce qu'il a un portefeuille qui joue sur les effets levier différents entre les actifs nationaux et domestiques ${ }^{13}$. Les investisseurs, coutumiers d'une situation qui a prévalu sous des noms divers pendant tout l'après-guerre, et rassurés par les cautions scientifiques fournis par les experts les plus cotés, se sont aisément laissés abuser par les connotations positives en anglais du terme securitization.

43 Si l'on délaisse l'art de la combinatoire pour examiner le constituant de base des titres ainsi diffusés, le terme le plus parlant est celui de subprime, qui est bien distribué comme un mot de la crise au sens où nous l'entendons, et qui participe à l'euphémisation générale.

\section{De l'euphémisation à l'inversion?}

Avant 2007, le terme subprime fait partie du jargon technique et il est pratiquement inconnu du public non spécialiste. À cette époque, investir dans un produit financier constitué de créances sur des ménages dont le credit rating est subprime ne laisse en rien présager une ruine prochaine. En effet, le néologisme est une litote très efficace qui fait écho au prime lending rate, c'est-à-dire, le taux consenti aux emprunteurs les plus sûrs par les autorités monétaires. En ce sens, être situé sous le niveau du prime (lending rate) signifie que l'on appartient au commun des mortels, ni plus ni moins. En réalité les ménages pudiquement répertoriés comme " au-dessous de l'optimum» (subprime household) sont souvent des NINJA, terme qui n'a pas connu plus de succès en France que les DINKs de la période faste dont ils sont l'exact antithèse ${ }^{14}$. 
Figure 13. - Subprime.

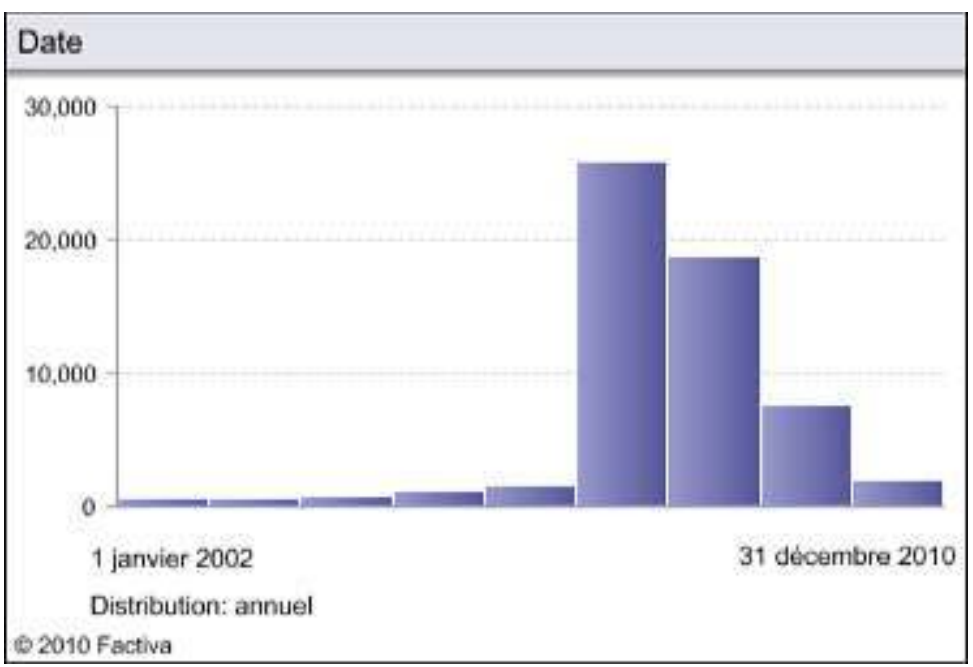

Ainsi, en 2007, le décodage s'effectue brutalement : les ménages en question apparaissent clairement insolvables dans le contexte du retournement du marché immobilier. Une créance subprime est une créance pourrie ${ }^{15}$, et tandis que tous les organismes financiers scrutent leurs bilans pour savoir s'ils en détiennent, le mot envahit la presse et les dépêches d'agences.

\section{La neutralité problématique du traducteur}

L'Amérique découvre que de nombreux ménages sont désormais under water ${ }^{16}$ et l'État fédéral nationalise de fait les deux plus grands organismes de garantie des prêts immobiliers (surnommés Fannie Mae et Freddie Mac). L'étape suivante, pour les ménages endettés, est la saisie immobilière, en anglais foreclosure ou repossession.

Figure 14. - Foreclosure / Repossession.

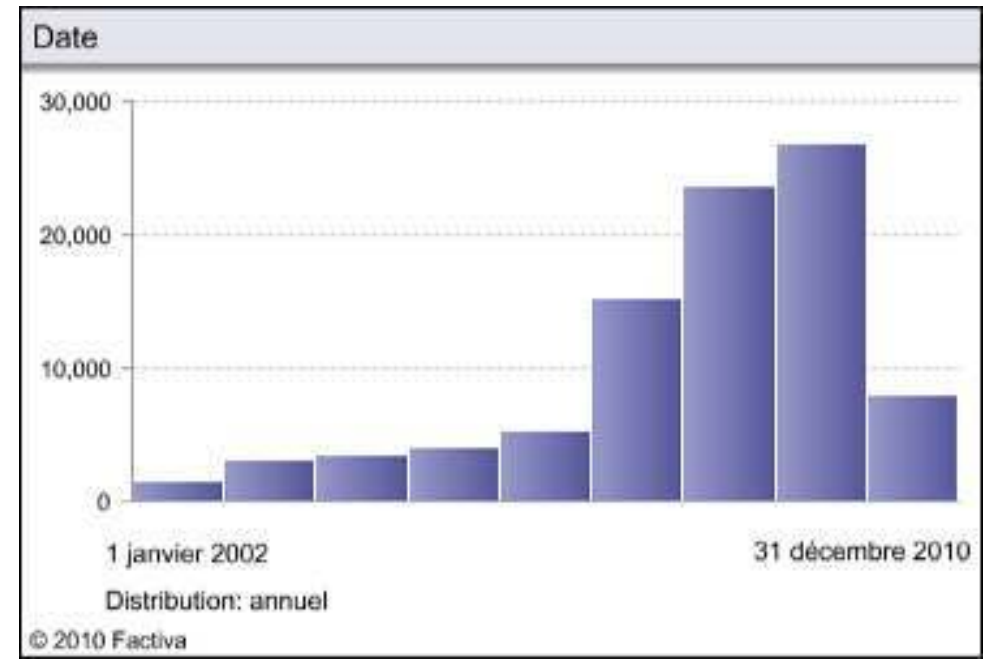

Le tableau 14 montre la distribution des expressions correspondant dans Factiva à la requête " Foreclos+ » et « repossess+ » pour recenser toutes les occurrences du substantif et des formes verbales. Le contradicteur pourrait penser tenir avec ces deux termes un 
contre-exemple. Loin d'euphémiser, les deux termes sont chargés d'intensité dramatique. Cependant, dans une très large mesure, foreclosure et repossession sont moins sinistres en anglais des États-Unis que le mot « saisie » en français avec toutes ses connotations dans le registre de la surprise et presque de la violence. Outre-Atlantique, ce qui est saisi en matière immobilière est rarement le salaire (l'arrêt-saisie est l'exception) mais le bien luimême sur lequel une hypothèque est prise, ce qui fait porter une part importante $d u$ risque de défaut de paiement sur la banque et non l'emprunteur. Plus euphémistique que rassurante en ce sens, la menace de la repossession participe malgré tout à l'inversion Orwellienne déjà identifiée, en nommant re-possession ce qui n'est en réalité rien d'autre qu'une dé-possession. Adopter le point de vue du créancier - sans vouloir pousser trop loin la démonstration - minore la violence faite au ménage expulsé et la rend presque légitime. Le banquier, pourtant n'est pas nécessairement blanchi par cette inversion de perspective.

Dans la quête du sens qui caractérise les communautés humaines, rien n'est plus convaincant qu'un récit dans lequel des forces positives et négatives sont en lutte.

Figure 15. - Bad bank.

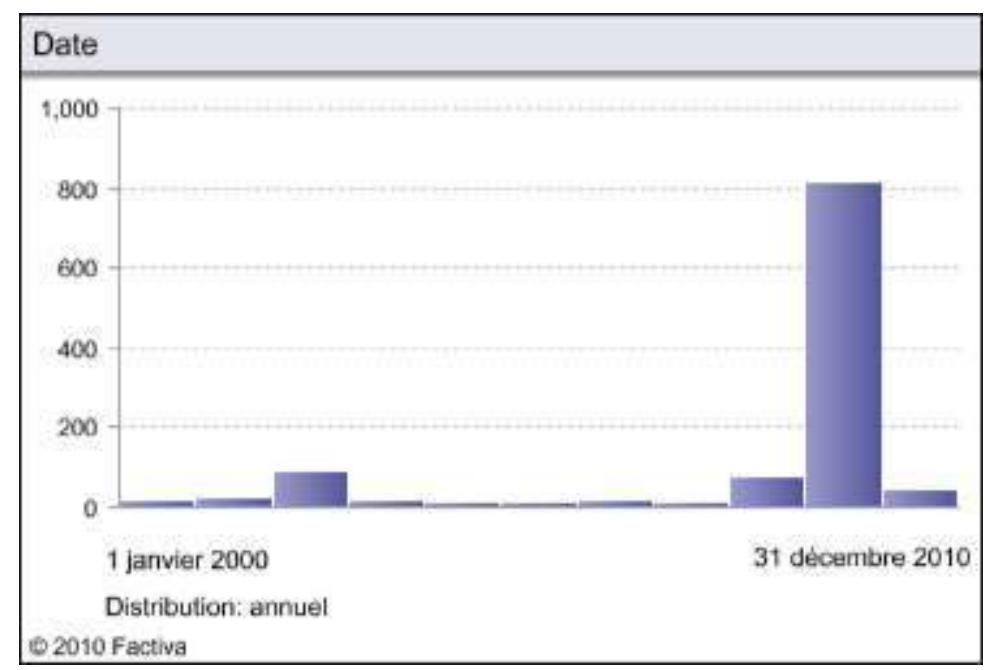
montage financier. Déjà dans l'explosion de la bulle des dot com en 2002 (figure 15), des bad banks, structures financières dont les actifs sont un concentré de créances douteuses, avaient permis d'apurer le bilan des autres banques. Les bad banks font la part du feu, et permettent par contraste aux autres banques d'apparaître comme des good banks, si elles ont survécu ${ }^{17}$. Pour désigner ce concept, le terme français, "structure de défaisance », a été forgé à cause de l'antécédent du Crédit Lyonnais, mais il n'a pas la force imaginaire de l'expression en anglais, et peut-être pour cette raison, il reste peu usité.

autant, nous ne sommes pas persuadés que l'expression bad bank renvoie nécessairement à un imaginaire manichéen teinté d'animisme qui donnerait naissance à l'intégrateur négatif du récit. Pour éviter toute surinterprétation, rappelons au lecteur qu'un chèque sans provision, en anglais américain courant, s'appelle un bad check, et que nous sommes alors plus près de l'incident de crédit que de l'Apocalypse.

51 Ces précautions énoncées, notons tout de même que le thème du gigantisme des banques revient très souvent enchâssé dans l'expression too big to fail, qui indique indirectement d'où provient le danger systémique. 
Figure 16. - FDIC - Federal Deposit Insurance Corporation.

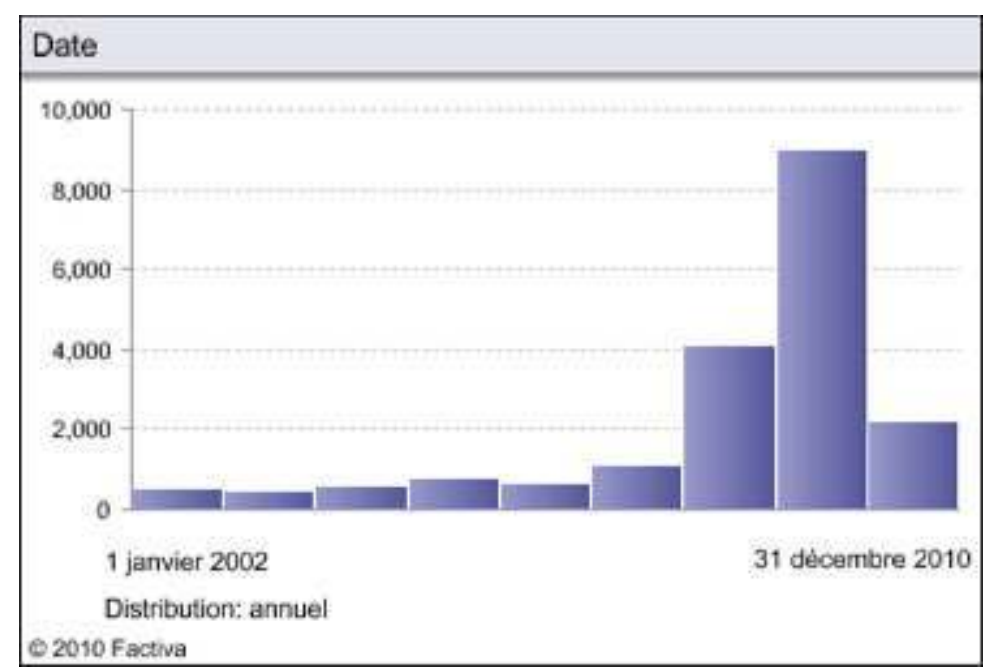
les années Reagan mais, ironie de l'histoire, c'est le démocrate Clinton qui signe en 1999 le Financial Services Modernization Act, menant ainsi à terme le démantèlement du GlassSteagall Act de $1933^{18}$ et levant les contraintes imposées par le Bank Holding Company Act de 1956.

Pourtant, les dispositions du New Deal ne disparaissent pas, comme l'illustre le cas du Federal Deposit Insurance Corporation (FDIC, figure 16), qui n'est assurément pas un mot de la crise de 2007-2009 mais de la Grande Dépression. Il faudrait pouvoir le tester sur la période 1933-1941, ce qui est hors des possibilités de la base de données Factiva pour démontrer que malgré un histogramme parfait sur la période 2002-2010, il appartient à une séquence historique beaucoup plus longue.

En revanche, le terme delinquency, qu'il faudrait traduire par "défaut de paiement" illustre bien comment un paramétrage précis de l'outil Factiva permet de retrouver la trace d'un phénomène qui, trop général pour caractériser la crise de 2007-2009, l'accompagne incontestablement. Comme le montre la figure 17, malgré la polysémie du terme ${ }^{19}$ testé, qui aurait pu produire des faux positifs, le fait d'avoir restreint la requête sur Factiva à la presse économique permet de mettre en évidence des occurrences qui cadrent bien avec la problématique étudiée. 
Figure 17. - Delinquency.

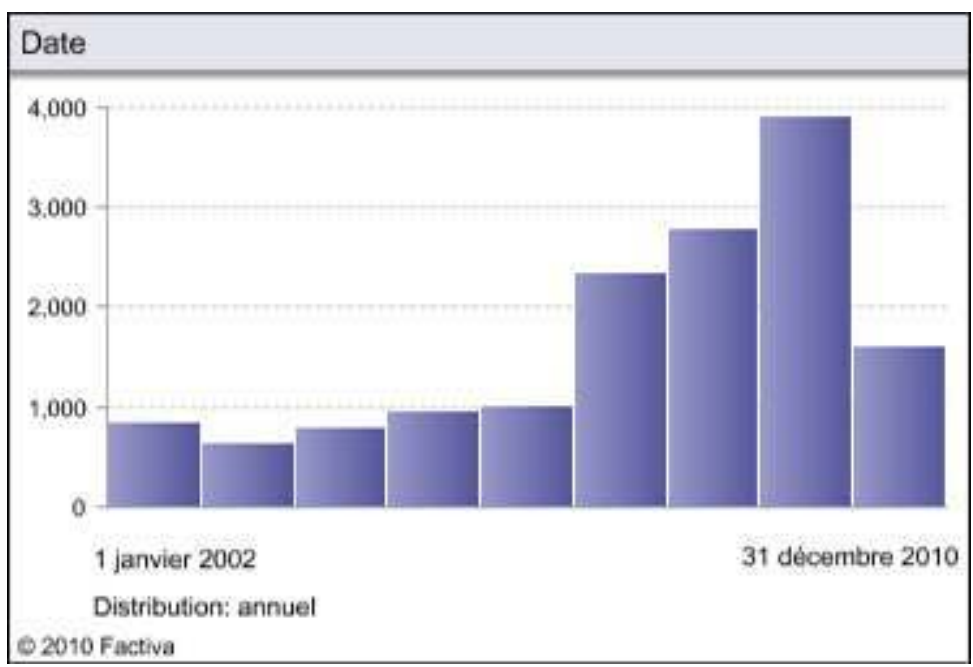
recovery plan) qui présente peut-être le profil de distribution le plus parfait pour « un mot de la crise ».

Le sigle n'apparaît pas avant 2008, car le plan en question est inauguré en 2008, ce qui signifie que les résultats très faibles entre 2000 et 2007 sont pratiquement tous des « faux positifs » dans la mesure où ils repèrent l'activité économique des entreprises fabriquant ou commercialisant des bâches (tarps forme abrégée de tarpaulins en anglais). Le profil est parfait, mais l'exploitation de l'expression, rarement évoquée et jamais traduite en français, est sans grand intérêt. Sauf, bien sûr, pour l'inconditionnel des connotations doté du sens de l'humour, qui ferait remarquer que les bâches imperméables protègent les chantiers, ou servent aux réparations de fortune après un ouragan.

Figure 18. - TARP - Troubled Assets Recovery Plan.

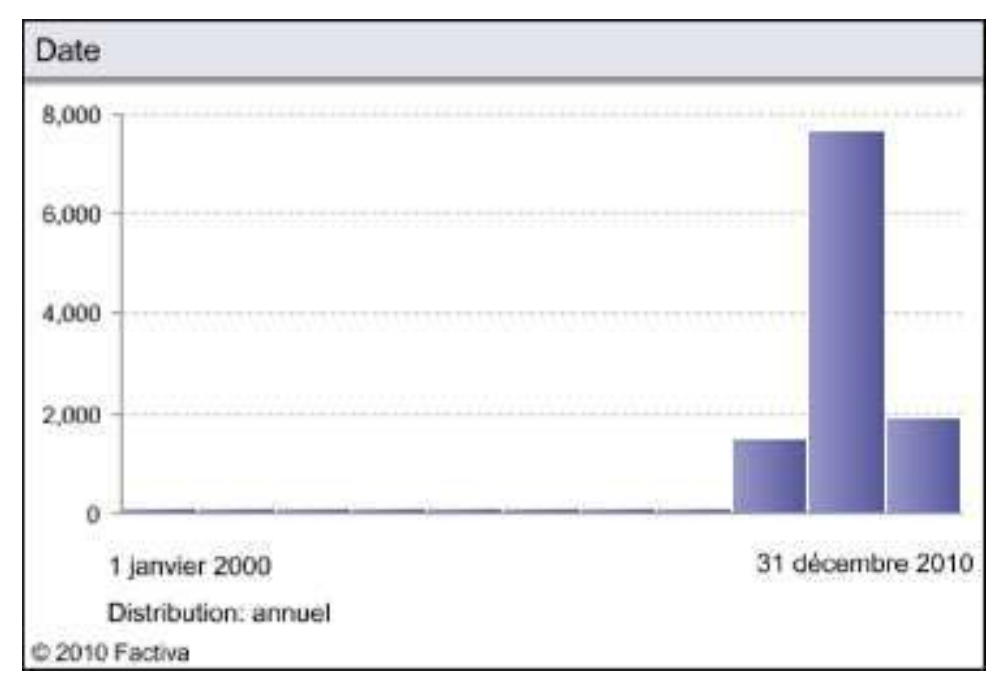

Plus sérieusement, c'est sur le terme hedge fund que nous amorcerons notre conclusion. Le terme n'est pas à proprement parler un mot de la crise, et le profil de l'histogramme qui lui correspond semble moins caractéristique que bien des autres profils déjà évoqués. 
Pourtant, avec ce terme nous touchons du doigt très clairement les enjeux de la traduction en français des mots de la crise.

Figure 19. - Hedge fund.

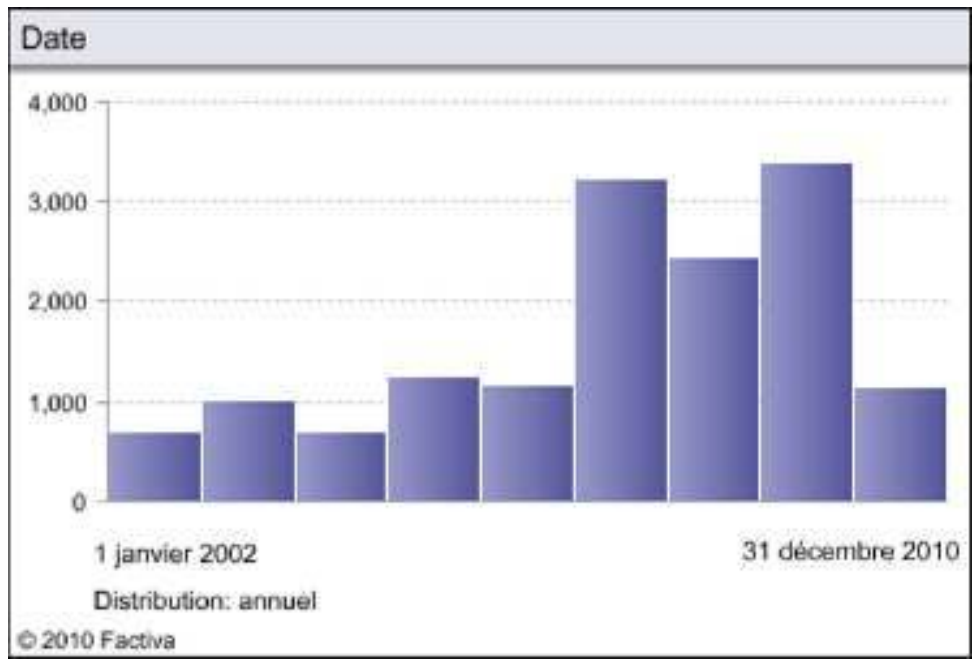
favorable au système économique libéral en recourant à l'euphémisation, à l'opacité, voire à l'inversion pure et simple. Remarquons d'ailleurs qu'euphémiser est un risque qui n'est jamais anodin, et qu'ici raisonner par degrés n'a pas de sens. Prétendre que l'on se borne à modaliser en présentant comme valeur sûre un placement hautement spéculatif n'est pas recevable. Pratiquer l'understatement pour décrire le risque encouru confine à la tromperie caractérisée. Le traducteur se trouve face à des responsabilités autant qu'à des problèmes techniques. C'est pourquoi l'attitude de neutralité apparente du technicien qui traduit sans expliciter finit par produire soit un langage codé réservé aux spécialistes en français, soit - cas extrême - une impasse sur la traduction et l'apport direct des mots étrangers. Dans ce cas, le traducteur refuse de dénoncer les conventions, comme le « banquier sans reproche » qui inspira à Keynes quelques lignes d'une ironie cinglante:

A sound banker, alas is not one who foresees danger and avoids it, but one who, when he is ruined, is ruined in a conventional way along with his fellows, so that no one can really blame him. (Keynes, 1931)

61 Après les accès "d'exubérance irrationnelle » du milieu des années 1990 (Greenspan, 1996), la fin de la première décennie du xxI siècle aura donc connu une «classique bulle 
euphorique » (Greenspan, 2010, p. 8 et suiv.) Malgré ces formules rassurantes, le bilan des pertes liées à la titrisation des crédits immobiliers est rapidement publié. Dès mars 2008, les chiffres du FMI mettent en évidence des pertes massives sur les produits dérivés dans les bilans bancaires ${ }^{20}$. Forts de la sagesse rétrospective que permet désormais la lecture des bilans comptables, les linguistes peuvent s'imaginer qu'ils ont percé à jour les fauxsemblants de l'euphémisation. Mieux encore, on pourrait penser que l'approche descriptive de la crise par le lexique n'est qu'un premier pas, et qu'une veille plus fine basée sur ces principes pourrait participer à l'élaboration de modèles prédictifs. Mais une telle démonstration des talents oraculaires des linguistes devra attendre la prochaine crise.

\section{BIBLIOGRAPHIE}

Fond Monétaire International, Regional Economic Outlook, Washington, D.C., 2008.

GOURINCHAS Pierre-Olivier et REY Hélène, « From World Banker to World Venture Capitalist: U.S. External Adjustment and the Exorbitant Privilege » [en ligne], dans R. H. Clarida (éd.), G7 Current Account Imbalances: Sustainabilityand Adjustment, University of Chicago Press, 2007. Disponible sur < http://www.nber.org/chapters/c0121> [article tiré d'une conférence de juin 2005].

GouRINCHAs Pierre-Olivier, Rey Hélène et Govillot Nicolas, Exorbitant Privilege and Exorbitant Duty, IMES Discussion Paper Series 2010-E-20, août 2010. Disponible sur <http://www.imes.boj>

KEYNES John Maynard, Consequences to the Banks of a Collpase in Money Values, 1931, cité en exergue dans H. M. Schwartz, Subprime Nation: American power, global capital, and the housing bubble, Cornell University Press, 2009.

KuHn Thomas S., The Structure of Scientific Revolutions, The University of Chicago Press, 1962.

SAMUELSON Paul, Economics, McGraw-Hill, 1973 [1953 - très nombreuses rééditions].

MARX Karl, The Capital, vol. III, 1894. Disponible depuis 1999 sur <http://marxists.org/> [rédigé en 1863-1883, inachevé et publié par F. Engels].

GREENSPAN Alan, « Remarks by Chairman Alan Greenspan At the Annual Dinner and Francis Boyer Lecture of The American Enterprise Institute for Public Policy Research, Washington, D.C., December 5, 1996 » [en ligne], 1996. Disponible sur <http://www.federalreserve.gov/ BOARDDOCS/SPEECHES/19961205.htm>

GREENSPAN Alan, « The mortgage market and consumer debt: Remarks by Chairman Alan Greenspan At America's Community Bankers Annual Convention Washington, D.C. », 19 octobre 2004. Disponible sur <http://www.federalreserve.gov/BOARDDOCS/Speeches/2004/20041019/ default.htm>

GREENSPAN Alan, « Economic flexibility;Remarks by Chairman Alan Greenspan Before the National Italian American Foundation, Washington, D.C., 12 octobre 2005. Disponible sur <http:// www.federalreserve.gov/boarddocs/speeches/2005/20051012/default.htm> GREENSPAN Alan, The Crisis, Greenspan Associates LLC, Second Draft : 9 mars 2010. 


\section{NOTES}

1. Le Groupe Multilingue de Recherche en Traduction Spécialisé (GREMUTS) est une des composantes de l'ILCEA. Il permet à des chercheurs spécialistes d'allemand, d'anglais, d'espagnol, d'italien et de russe de travailler ensemble sur des problématiques transversales.

2. Cette étude n'est pas un article d'économie: elle ne prend pas parti dans le débat - entre libéraux et keynésiens pour ne citer que ces deux écoles - sur les causes de la crise ou les remèdes souhaitables. Les mécanismes précis seront décrits a minima à l'appui des questions de traduction.

3. Selon Thomas Kuhn, le paradigme est un ensemble de théories qui emporte l'adhésion durable des penseurs et des praticiens scientifiques, un système cohérent mais assez ouvert pour intégrer les évolutions de la pensée. La crise de 2008 semblait avoir disqualifié le paradigme libéral et réhabilité l'approche des Keynésiens, régulateurs ou institutionnalistes, mais nous manquons du recul nécessaire notamment pour juger des résultats de la relance "non-orthodoxe" orchestrée par la FED aux États-Unis en 2010-2011.

4. Les théories cycliques sont dans tous les manuels d'économie depuis les années 1930. Le classement de Kondratieff à Kitchin correspond à des cycles de plus en plus courts, de 50 ans ou plus à 2 ou 3 ans : voir le plus célèbre et le plus répandu des manuels américains celui de Samuelson, chapitre 14, p. 249 et suiv. essentiellement sur Kondratieff et Kutznets. L'édition de 1973 - en plein consensus Keynésien et avant le premier choc pétrolier - émet l'idée que les cycles sont désormais sous contrôle.

5. L'économie est généralement déclarée en récession quand le taux de croissance demeure négatif pendant deux trimestres consécutifs; le terme dépression est réservé à des périodes longues et aux baisses de forte amplitude.

6. Nous faisons évidemment ici allusion à l'école marxiste. Le tome III du Capital est consacré presque en entier aux phénomènes non cycliques comme la loi de la baisse tendancielle du profit (chapitre 13) qui exacerbent progressivement les contradictions internes du système et conduisent à la révolution prolétarienne.

7. La plus ancienne allusion au cycle économique - dans ce cas de 14 ans - se trouve en effet dans la Bible (Genèse 41, 1-36).

8. Un « faux positif » est un artéfact qui produit un résultat positif à un test alors que le véritable résultat est négatif. Voir infra le cas du mot tarp(aulin) (une bâche) et du sigle TARP (troubled assets recovery plan).

9. L'observation d'une symétrie axiale serait moins évidente - d'où la rotation du graphique.

10. Le principe désigné par non-recourse pousse à l'extrême la logique hypothécaire. La restitution du bien sur lequel l'hypothèque est prise (en rendant «les clés à la banque " selon l'expression consacrée) éteint la créance selon un mécanisme qui rappelle celui de la responsabilité limitée dans le droit des affaires.

11. Greenspan s'était déjà illustré par un discours de 1996 qui se voulait rassurant mais qui contenait l'expression irrational exuberance, devenue célèbre depuis l'effondrement boursier qui avait suivi (Greenspan, 1996).

12. Terme non traduit, ou dont la traduction proposée n'a pas franchi les limites des spécialistes.

13. Gourinchas et Rey élaborent leur article en 2005 soit bien avant le déclenchement de la crise. Il sera publié en 2007 et mis à jour après notre période (Gourinchas, Rey et Govillot, 2010). Ils décrivent la position des États-Unis comme celle d'un investisseur: " the net foreign asset position of a country is nothing but a leveraged portfolio where the country is short in domestic assets and long in foreign assets "

14. DINKs : double income no kids - NINJA : no income, no job nor assets. 
15. Le terme junk bond avait au moins le mérite de la clarté.

16. Terme qui n'est pas testable par Factiva, « la tête sous l'eau » : se dit des emprunteurs dont le bien immobilier a moins de valeur que le montant du prêt restant à rembourser.

17. Nombre de petites banques font faillite aux États-Unis pendant la période, mais l'effondrement de Lehman Brothers, suivi du sauvetage de l'American Insurance Group (qui n'est pas techniquement un établissement bancaire), opère comme l'accélérateur de la crise boursière. 18. Héritage du New Deal, le Banking Act de 1933 - nom officiel- jetait les bases d'une séparation entre banque de dépôt et banque d'affaires, pour mettre les petits épargnants à l'abri des faillites bancaires. Dans la foulée le FDIC fut également créé (voir infra).

19. Le terme le plus fréquent pour "délinquence » demeure crime et non delinquency - qui, s'il existe s'utilise davantage dans le contexte de «juvenile delinquency »- ce qui limite en tout état de cause les faux positifs.

20. FMI, 2008, p. 25, tableau 5. Les chiffres sont déjà, en 2008, de 123 milliards de dollars en Europe et 144 aux USA - en ne parlant que des valeurs effectivement reportées dans le bilan des banques pour des instruments dérivés les plus «innovants» du type ABS (asset-backed securities), CDOs (Collaterilized debt obligations), etc.

\section{RÉSUMÉS}

L'article s'attache en premier à vérifier graphiquement qu'il existe, pour caractériser la crise économique de 2007-2009 (phénomène cyclique), un lexique caractéristique relativement inédit pour décrire les phénomènes survenus. Les termes anglais, les "mots de la crise" originaux, une fois testés à l'aide de la base de données Factiva, seront exploités par les spécialistes des autres langues dans une optique de traductologie. Le second objectif de l'étude est de rendre compte de la non-traduction d'une grande partie de ces termes en français par une réflexion sur les enjeux de traduction spécifiques à ce lexique spécialisé, caractérisé par l'euphémisation, voire l'inversion des signifiés.

This paper studies graphs to check the existence of a lexicon specifically designed to describe the economic crisis of 2007-2009. The English terms - the original "words of the crisis" - are tested through the Factiva database and subsequently analysed by researchers of other languages specializing in translation studies. The second objective of this study deals with the many terms not translated into French and analyses what is at stake for translators dealing with a type of specialized lexicon characterized by understatements and, in some cases, inverted meanings.

\section{INDEX}

Keywords : 2007-2009, bad bank, bubble, economic crisis, economics-related translation, hedge fund, mortgage, recession, securitization, specialized economic lexicon, subprime, toxic assets Mots-clés : 2007-2009, actifs toxiques, bad bank, bulle, crise économique, fond spéculatif, lexique économique spécialisé, prêt immobilier, récession, subprime, titrisation, traduction économique 


\section{AUTEUR}

\section{CHRISTIAN LEBLOND}

MCF, Université Stendhal - Grenoble 3. ILCEA 